\title{
Fluctuation and dissipation of axial charge from massive quarks
}

\author{
De-fu Hou ${ }^{1, *}$ and Shu $\mathrm{Lin}^{2, \dagger}$ \\ ${ }^{1}$ Institute of Particle Physics (IOPP) and Key Laboratory of Quark and Lepton Physics (MOE), \\ Central China Normal University, Wuhan 430079, China \\ ${ }^{2}$ School of Physics and Astronomy, Sun Yat-Sen University, Zhuhai, 519082, China
}

(Received 24 May 2018; published 12 September 2018)

\begin{abstract}
In quantum chromodynamics, axial charge is known to be nonconserved due to chiral anomaly and nonvanishing quark mass. In this paper, we explore the role of quark mass in axial charge fluctuation and dissipation. We present two separate calculations of the axial charge correlator, which describe dynamics of axial charge. The first is free quarks at a finite temperature. We find that axial charge can be generated through effective quantum fluctuations in free theory. However, the fluctuation does not follow a random walk behavior. Because of the presence of an axial symmetry breaking mass term, the axial charge also does not settle asymptotically to the thermodynamic limit given by susceptibility. The second calculation is in a weakly coupled quark gluon plasma. We find in the hard thermal loop (HTL) approximation, the quarkgluon interaction leads to random walk growth of the axial charge, but dissipation is not visible. We estimate the relaxation timescale for the axial charge, finding it lies beyond the HTL regime.
\end{abstract}

DOI: 10.1103/PhysRevD.98.054014

\section{INTRODUCTION}

The chiral anomaly is one of the most intriguing discoveries in quantum field theory. Over the past ten years, its manifestations in macroscopic phenomena such as the chiral magnetic effect and chiral vortical effect have triggered significant interest across different communities [1-6]. There have been continuous efforts in searching for chiral magnetic effect and chiral vortical effect in the quark gluon plasma produced in heavy ion collisions [7-9], as well as in Weyl semimetal $[10,11]$. For comprehensive reviews of current status, we refer to [12-14]. Effective descriptions of chiral magnetic effect and chiral vortical effect have been developed for chiral fermions, including anomalous hydrodynamics [15-17], chiral kinetic theory [18-24], and holography [25-30]. Both theoretical frameworks reveal beautiful structures in the chiral limit. In most phenomenological applications of the two frameworks, axial charge density is needed as an input, usually modeled by axial charge chemical potential $\mu_{5}$. Possible issues with using spacetime dependent $\mu_{5}$ are pointed out by one of the authors [31]. To use $\mu_{5}$ properly, a better understanding of the dynamics of axial charge is needed.

\footnotetext{
*houdf@mail.ccnu.edu.cn

†linshu8@mail.sysu.edu.cn
}

Published by the American Physical Society under the terms of the Creative Commons Attribution 4.0 International license. Further distribution of this work must maintain attribution to the author(s) and the published article's title, journal citation, and DOI. Funded by SCOAP ${ }^{3}$.
One of the well-known generation mechanisms of axial charge is through a topological fluctuation of the gluon field $[32,33]$. Because of the fluctuation-dissipation theorem, this mechanism can also cause damping of the axial charge. Most phenomenological studies ignore the damping effect in the dynamics of axial charge. The interplay of generation and damping is known to lead to interesting dynamics of axial charge [34]. In addition to topological fluctuation of gluons, fermion mass violates axial charge conservation explicitly. Questions on the role of fermion mass also arises in a different context. On the theoretical side, any fundamental fermion is known to carry mass. Knowing how fermion mass modifies the existing frameworks is a key ingredient. On the phenomenological side, quantifying the magnitude of the mass effect is needed for reliable modeling. The damping effect is first discussed in [35] for an electron in the neutron star. The generation effect is proposed by one of the authors [36,37] for the supersymmetric gauge theory based on a holographic model.

This paper aims at providing a unified description of the two effects in the same setting. To be specific, we study dynamics of total axial charge in QCD in the weakly coupled regime, where perturbative calculation is possible. The results apply equally well to QED. For pedagogical reasons, we begin with axial charge dynamics in free quark theory in Sec. II. We then move on to carry out the same study in weakly coupled QCD plasma in Sec. III. We find that unlike free theory, weakly coupled plasma can generate axial charge through a quark mass term similar to the topological fluctuation of gluons. The rate of generation, to 
be coined mass diffusion rate, is numerically much smaller than the topological fluctuation rate at physically relevant coupling for a strange quark mass. We summarize in Sec. IV.

\section{AXIAL CHARGE DYNAMICS IN FREE THEORY}

For simplicity, we first study axial charge dynamics in free theory. This allows us to set the stage and gain insights into the dynamics. We first note that classical fermions satisfying the Dirac equation do not have a net axial charge, even though axial symmetry is broken by fermion mass. In order to generate the net axial charge, we need the quantum fluctuation to push fermions off shell. The quantity characterizing axial charge dynamics is the following Wightman correlator:

$$
\int d t d^{3} \mathbf{x} e^{i q_{0} t}\left\langle\psi^{+} \gamma^{5} \psi(t, x) \psi^{+} \gamma^{5} \psi(0)\right\rangle
$$

This describes dynamics of total axial charge $N_{5}=\int d^{3} \mathbf{x} \psi \psi^{+} \gamma^{5} \psi(x)$. The contribution to (1) comes from a simple quark loop diagram. The contribution is given by

$$
G^{>}(Q)=\int \frac{d^{4} K}{(2 \pi)^{4}} \operatorname{Tr} S_{21}(K) S_{12}(K-Q),
$$

with $K=\left(k_{0}, \vec{k}\right)$ and $Q=\left(q_{0}, 0\right)$. Let us first consider the case $q_{0}>0$. Using delta functions in fermion propagators $S_{21}$ and $S_{12}$, we find the only possible kinematics is $k_{0}=E_{k}$, and $k_{0}-q_{0}=-E_{k}$, with $E_{k}=\sqrt{k^{2}+m^{2}}$. It is not difficult to evaluate the integral to obtain

$$
\begin{aligned}
G^{>}\left(q_{0}\right)= & \frac{N_{f} N_{c}}{\pi} \tilde{f}\left(-\frac{q_{0}}{2}\right)^{2} \sqrt{\left(\frac{q_{0}}{2}\right)^{2}-m^{2}} \frac{2 m^{2}}{q_{0}} \theta\left(q_{0}-2 m\right), \quad q_{0}>0, \\
& \text { with } \tilde{f}\left(-q_{0} / 2\right)=\frac{1}{e^{-\beta q_{0} / 2}+1} .
\end{aligned}
$$

This gives the spectral function $\rho\left(q_{0}\right)$,

$$
\begin{aligned}
\rho\left(q_{0}\right)= & \frac{G^{>}\left(q_{0}\right)}{1+f\left(q_{0}\right)}=\frac{N_{f} N_{c}}{\pi} \frac{\tilde{f}\left(q_{0} / 2\right)}{f\left(q_{0} / 2\right)} \sqrt{\left(\frac{q_{0}}{2}\right)^{2}-m^{2}} \frac{2 m^{2}}{q_{0}} \theta\left(q_{0}-2 m\right), \quad q_{0}>0, \\
& \text { with } \quad f\left(q_{0} / 2\right)=\frac{1}{e^{\beta q_{0} / 2}-1} .
\end{aligned}
$$

For the case $q_{0}<0$, we use the representation $G^{>}\left(q_{0}\right)=$ $\rho\left(q_{0}\right)\left(1+f\left(q_{0}\right)\right)$ and the property $\rho\left(-q_{0}\right)=-\rho\left(q_{0}\right)$ to obtain

$$
\begin{aligned}
G^{>}\left(q_{0}\right) & =-\frac{N_{f} N_{c}}{\pi} \tilde{f}\left(-\frac{q_{0}}{2}\right)^{2} \\
& \times \sqrt{\left(\frac{q_{0}}{2}\right)^{2}-m^{2}} \frac{2 m^{2}}{q_{0}} \theta\left(-q_{0}-2 m\right), \quad q_{0}<0 .
\end{aligned}
$$

The above evaluation misses a contribution proportional to $\delta\left(q_{0}\right)$. It is known that such a contribution corresponds to susceptibility for a conserved charge [38]. We can include this contribution by assuming the following decomposition of $G^{>}$:

$$
G^{>}=G\left(q_{0}, T, m\right)+\delta\left(q_{0}\right) F(T, m) .
$$

We have already obtained $G\left(q_{0}, T, m\right)$ in (3) and (5). $F(T, m)$ can be obtained by

$$
F(T, m)=\lim _{\epsilon \rightarrow 0} \int_{-\epsilon}^{\epsilon} d q_{0} G^{>}\left(q_{0}, T, m\right) .
$$

Using (2) for the evaluation of (7), we obtain

$$
F(T, m)=\int_{0}^{\infty} d k \frac{4}{\pi} \frac{k^{4}}{E_{k}^{2}} \tilde{f}\left(E_{k}\right)\left(1+\tilde{f}\left(E_{k}\right)\right)
$$

It is instructive to compare (6) with the Wightman correlator for $N=\int d^{3} \mathbf{x} \psi^{+} \psi(x)$. Following a similar procedure, we would obtain the same expression (6) but without $G\left(q_{0}, T, m\right)$. It is known that for conserved charge $N, G^{>}=2 \pi T \delta\left(q_{0}\right) \chi$, and thus $F(T, m)$ is simply related to susceptibility $F(T, m)=2 \pi T \chi$. By analogy, we define the susceptibility of $N_{5}$ from the contribution of $F$ by $\chi=F /(2 \pi T)$. We plot the $m$ dependence of $\chi$ in Fig. 1 .

The fluctuation of $N_{5}$ is characterized by the correlator $\left\langle\Delta N_{5}(t)^{2}\right\rangle \equiv\left\langle\left(N_{5}(t)-N_{5}(0)\right)^{2}\right\rangle$, which can be expressed by $G^{>}$as 


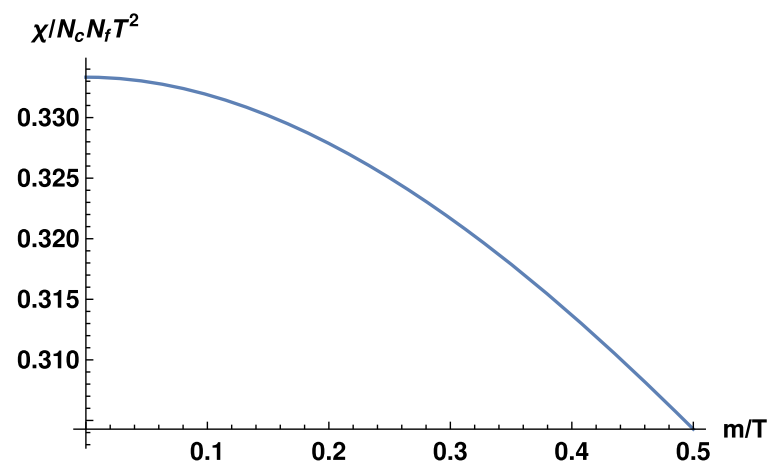

FIG. 1. Normalized susceptibility versus $m / T$ for both $N_{5}$ and $N$. It reduces to known result $\chi=N_{c} N_{f} T^{2} / 3$ in the massless limit.

$$
\left\langle\Delta N_{5}(t)^{2}\right\rangle=V \int \frac{d q_{0}}{2 \pi}\left(2-e^{i q_{0} t}-e^{-i q_{0} t}\right) G^{>}\left(q_{0}\right),
$$

with $V=\int d^{3} x$ being the volume factor. Let us look at the contribution from $F$ and $G$ separately. The evaluation of the former is subtle: a naive integration of $\delta\left(q_{0}\right)$ gives a vanishing result. However, on general ground we expect as $t \rightarrow \infty$

$$
\begin{aligned}
\left\langle\Delta N_{5}(t)^{2}\right\rangle= & \left\langle N_{5}(t)^{2}\right\rangle+\left\langle N_{5}(0)^{2}\right\rangle-\left\langle N_{5}(t) N_{5}(0)\right\rangle \\
& -\left\langle N_{5}(0) N_{5}(t)\right\rangle \rightarrow 2\left\langle N_{5}(0)^{2}\right\rangle,
\end{aligned}
$$

where we used $\left\langle N_{5}(t) N_{5}(0)\right\rangle \rightarrow 0$ and $\left\langle N_{5}(t)^{2}\right\rangle=$ $\left\langle N_{5}(0)^{2}\right\rangle{ }^{1}$ Taking the contribution to $\left\langle N_{5}(0)^{2}\right\rangle$ from $F$, we would instead obtain

$$
\left\langle\Delta N_{5}(t)^{2}\right\rangle_{F}=2 \chi T V
$$

The origin of the disagreement is that the two limits $q_{0} \rightarrow 0$ (or $t \rightarrow \infty$ ) and $k \rightarrow 0$ (or $V \rightarrow \infty$ ) do not commute. In (9), we take $V \rightarrow \infty$ first while in (10), we take $t \rightarrow \infty$ first. Physically they are not equivalent: since a conserved charge can fluctuate only through a charge exchange with a heat bath, taking $V \rightarrow \infty$ requires a larger and larger heat bath, and consequently a longer and longer equilibration time. To reproduce (10), we should take the limit $t \rightarrow \infty$ first, which amounts to dropping the rapid oscillating terms in (9). The resulting $\left\langle\Delta N_{5}(t)^{2}\right\rangle$ indeed obtain (10).

Now we turn to the evaluation of the contribution from $G$. This is intrinsic to the breaking of the axial symmetry. It corresponds to fluctuation by itself, not relying on a charge exchange with a heat bath. It is easy to see from (3) that the fluctuation also exists in vacuum. Plugging (3) and (5) into (9), we find the integral contains a UV divergence. We regularize by subtracting the vacuum contribution:

\footnotetext{
${ }^{1}$ In [34], the same quantity is calculated in the stochastic hydrodynamics framework. $\chi T V$ is obtained instead. The reason is we set initial $N_{5}(0)=0$. This amounts to subtracting a baseline for the fluctuation.
}

$$
\begin{aligned}
\left\langle\Delta N_{5}(t)^{2}\right\rangle_{G}= & V N_{f} N_{c} \int_{0}^{\infty} \frac{d q_{0}}{2 \pi} \frac{2\left(1-\cos \left(q_{0} t\right)\right)}{\pi \hbar^{3}} \\
& \times\left[\frac{\tilde{f}\left(q_{0} / 2\right)^{2}}{\tilde{f}\left(q_{0}\right)}-1\right] \sqrt{\left(\frac{q_{0}}{2}\right)^{2}-m^{2}} \\
& \times \frac{m^{2}}{q_{0}} \theta\left(q_{0}-2 m\right) .
\end{aligned}
$$

We have restored the factor of $\hbar$ in (12). Note that on the left-hand side, $N_{5}$ is dimensionless. On the right-hand side, the dimension reads $(\text { energy })^{3}(\text { length })^{3} / \hbar^{3}$, also dimensionless. We point out two counterintuitive features of (12). The fluctuation of $N_{5}$ contains the explicit factor of $\hbar$, indicating it is a consequence of quantum fluctuation. However, we know in the free quark case there is no interaction to induce quantum fluctuation. The other odd feature is that the regularized fluctuation is negative (as is clear from the negativity of the square bracket). It means that the fluctuation at finite temperature is smaller compared to that in the vacuum.

The two seemingly odd features are, in fact, related: Although quarks are free at the Lagrangian level, FermiDirac statistics obeyed by quarks in equilibrium provides effective interaction; thus quantum fluctuation is present. Furthermore, this also gives a quantitative explanation of the negative sign in the regularized fluctuations. The effect of Fermi-Dirac statistics becomes prominent as we lower the temperature. In the vacuum case, quantum fluctuation is maximal, and thus the vacuum fluctuation is larger than any finite temperature fluctuation, giving rise to negative regularized fluctuation. Equation (12) can be evaluated numerically. We include the time evolution of (12) for different $m$ in Fig. 2. The fluctuation is characterized by an initial rise followed by oscillatory decay to asymptotic value. Figure 2 suggests the initial rise satisfies the scaling $\left\langle\Delta N_{5}(t)^{2}\right\rangle \propto m^{2}$. Since the mass term is the source of

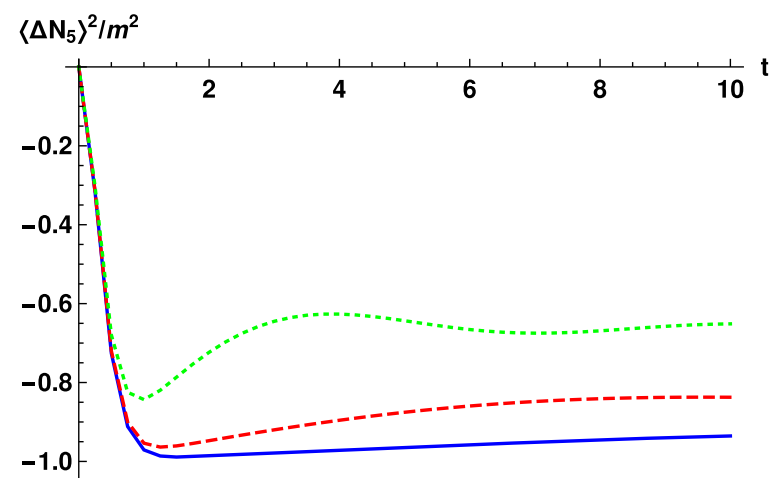

FIG. 2. Contributions from intrinsic fluctuation $\left\langle\Delta N_{5}(t)^{2}\right\rangle / m^{2}$ for different masses: blue solid line for $m=1 / 10$, red dashed line for $m=1 / 5$, and green dotted line for $m=1 / 2$. The unit is set by $T=1$. The fluctuation is characterized by an initial rise followed by oscillatory decay to asymptotic value. The case with a larger mass shows more rapid convergence to the asymptotic value. 
fluctuation, the $m^{2}$ dependence as the lowest order expansion is expected from analyticity in $m$. Nonanalyticity can occur in the presence of the external field due to the Schwinger effect [5,39]. Furthermore, if we regard the period of oscillation as relaxation time, Fig. 2 also implies a shorter relaxation time at a larger mass, which is consistent with the expectation on general grounds.

To summarize, we find the fluctuation of $N_{5}$ contains two contributions (10) and (12):

$$
\left\langle\Delta N_{5}(t)^{2}\right\rangle=2 V \chi T+V \int \frac{d q_{0}}{2 \pi}\left(2-e^{i q_{0} t}-e^{-i q_{0} t}\right) G\left(q_{0}\right),
$$

where in the second line the limit $t \rightarrow \infty$ should be taken. The $\chi$ term arises from a charge exchange with a heat bath. The term proportional to $G$ is intrinsic to the breaking of the axial symmetry. It exists without a heat bath. We could have view the second term as a correction to susceptibility, but this interpretation is misleading. Note that the second term is not necessarily proportional to temperature as it arises from quantum fluctuation. In the next section, we will focus on the modification of the intrinsic fluctuation by interaction.

\section{AXIAL CHARGE DYNAMICS IN WEAKLY COUPLED QGP}

After the warm-up, we move on to the calculation in weakly coupled quark gluon plasma (QGP). We expect the same structure of the Wightman correlator as (6). The term proportional to $F$ is related to the susceptibility of $N$. It has been calculated in perturbation theory [40]. The other term is entirely due to the nonconservation of $N_{5}$. We will calculate $G_{G}^{>}$in weakly coupled QGP, with the subscript indicating it only contains the $G$ term. We start with the retarded correlator, whose imaginary part is related to the Wightman correlator,

$$
G^{R}\left(q_{0}\right) \equiv \int d t d^{3} \mathbf{x} e^{i q_{0} t}\left\langle\left[\psi^{+} \gamma^{5} \psi(x), \psi^{+} \gamma^{5} \psi(0)\right]\right\rangle .
$$

We will proceed in imaginary time formalism and analytically continue to real time in the end. We work in the hard thermal loop (HTL) approximation at one loop order. It is known from the calculation of susceptibility that the one loop result of HTL is not complete ([40] and references therein). However, the main purpose of this paper is to demonstrate the diffusive behavior of the axial charge from the quark mass effect; we restrict ourselves to one loop order and leave more refined studies for future work.

At one loop order, the $N_{5}$ correlator receives contributions from three diagrams as shown in Fig. 3. The first diagram contains a soft quark loop with pseudophotonquark vertices $(\tilde{\gamma} q q)$. The second diagram contains a soft gluon loop with two-pseudophoton-two-gluon vertex $(2 \tilde{\gamma} 2 g)$, and the third diagram contains a quark loop with a two-pseudophoton-two-quark vertex $(2 \tilde{\gamma} 2 q)$ vertex. Here we used $\tilde{\gamma}$ to denote the pseudophoton leg. These resummed vertices are to be evaluated for HTL diagrams. When the quark mass $m=0$, we can easily show by commuting $\gamma^{5}$ with $\gamma^{\mu}$ the following relations:

$$
\begin{aligned}
\tilde{\gamma} q q & =\gamma q q \times \gamma^{5}, \\
2 \tilde{\gamma} 2 g & =2 \gamma 2 g, \\
2 \tilde{\gamma} 2 q & =2 \gamma 2 q .
\end{aligned}
$$

We have used schematic notations. The first line of (15) means the pseudophoton-quark vertex equals photon-quark vertex times $\gamma^{5}$ and similarly for the second and third equalities. When $m \neq 0$, in general all the vertices involving the pseudophoton receive corrections from $m$. To simplify the computation, we take the quark mass to be soft, i.e., $m \sim g T$. We stress that this is the "current quark mass," not to be confused with the thermal quark mass, which will appear below as $m_{f}$. The current quark mass itself should be $T$ independent. The relation $m \sim g T$ is only meant for numerical values for specific $m, g$, and $T$.

In the spirit of HTL, we will drop any contributions at $O\left(\frac{m^{2}}{T^{2}}\right)$. This allows (15) to hold in this approximation. We are ready to write down explicit expressions of the vertices involved,

$$
\begin{aligned}
& { }^{*} \Gamma_{5 \mu}\left(P_{1}, P_{2}\right)=\left(\gamma_{\mu}-m_{f}^{2} \int \frac{d \Omega}{4 \pi} \frac{\hat{K}_{\mu} \hat{K}}{\left(P_{1} \cdot \hat{K}\right)\left(P_{2} \cdot \hat{K}\right)}\right) \gamma_{5}, \\
& { }^{*} \Gamma_{\mu \nu}\left(P_{1}, P_{2}, Q_{1}, Q_{2}\right) \\
& =-m_{f}^{2} \int \frac{d \Omega}{4 \pi} \frac{\hat{K}_{\mu} \hat{K}_{\nu} \hat{K}}{\left(P_{1}+Q_{1}\right) \cdot \hat{K}\left(P_{2}-Q_{1}\right) \cdot \hat{K}} \\
& \quad \times\left[\frac{1}{P_{1} \cdot \hat{K}}+\frac{1}{P_{2} \cdot \hat{K}}\right],
\end{aligned}
$$

with $P_{1}$ and $P_{2}$ being quark momenta and $Q_{1}$ being one of the gluon momenta. $m_{f}^{2}=\frac{1}{8} C_{F} g^{2} T^{2}$ is the thermal quark mass, not to be confused with the current quark mass $m$.
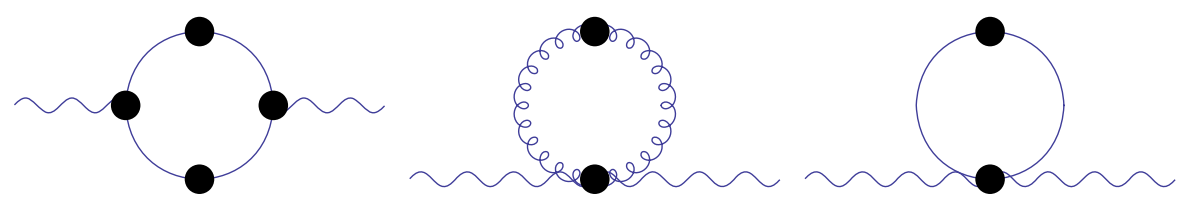

FIG. 3. Three leading one loop diagrams contributing to (14) in the HTL approximation. 
The null vector is defined as $\hat{K}=(-i, \hat{k})$. The remaining $2 \tilde{\gamma} 2 g$ vertex is obtainable by sending two generators to 1 in four-gluon vertex. This leads to a vanishing result, and thus the second diagram drops out. Unlike vertices, the resummed quark propagator does get modification due to quark mass as follows:

$$
\begin{aligned}
{ }^{*} S(P) & =\frac{1}{\not P+\Sigma+m} \\
& =\frac{\frac{1}{2}\left(\Delta_{+}+\Delta_{-}\right) i \gamma_{4}+\frac{1}{2}\left(\Delta_{+}-\Delta_{-}\right)-m \Delta_{+} \Delta_{-}}{1-m^{2} \Delta_{+} \Delta_{-}}
\end{aligned}
$$

with $\gamma_{p}=\hat{p} \cdot \vec{\gamma}$ and

$$
\begin{aligned}
\Sigma & =\frac{m_{f}^{2}}{p}\left[i \gamma_{4} Q_{0}\left(\frac{\omega}{p}\right)+\gamma_{p}\left(1-\frac{i \omega}{p} Q_{0}\left(\frac{\omega}{p}\right)\right)\right], \\
\frac{1}{\Delta_{ \pm}(P)} & =i \omega \mp p-\frac{m_{f}^{2}}{p}\left[Q_{0}\left(\frac{i \omega}{p}\right) \mp Q_{1}\left(\frac{i \omega}{p}\right)\right] .
\end{aligned}
$$

Our calculation heavily relies on Ward identities. We note the $\gamma q q$ vertex and the $2 \gamma 2 q$ vertex satisfy the following Ward identities:

$$
\begin{aligned}
Q_{1 \mu}{ }^{*} \Gamma_{\mu \nu}\left(P_{1}, P_{2}, Q_{1}\right) & =\Gamma_{\nu}\left(P_{1}, P_{2}-Q_{1}\right)-\Gamma_{\nu}\left(P_{1}+Q_{1}, P_{2}\right), \\
\left(P_{1}-P_{3}\right)_{\mu}{ }^{*} \Gamma_{\mu}\left(P_{1}, P_{3}\right) & =\Sigma\left(P_{1}\right)-\Sigma\left(P_{3}\right) .
\end{aligned}
$$

For our purpose, we take quark momenta as $P_{1}=P_{2}=P$, $P_{3}=P^{\prime}$, and pseudophoton momentum $Q=(-\varpi, \mathbf{0})=$ $P-P^{\prime}$. Consequently ${ }^{*} \Gamma_{44}$ and ${ }^{*} \Gamma_{4}$ can be uniquely fixed by Ward identities as

$$
\begin{aligned}
-\varpi^{*} \Gamma_{44}(P, P, Q) & ={ }^{*} \Gamma_{4}(P, P-Q)-{ }^{*} \Gamma_{4}(P+Q, P), \\
-\varpi^{*} \Gamma_{4}\left(P, P^{\prime}\right) & =\Sigma(P)-\Sigma\left(P^{\prime}\right) .
\end{aligned}
$$

We proceed by evaluating the tadpole diagram

$$
\int \frac{d^{4} p}{(2 \pi)^{4}} \operatorname{tr}^{*} \Gamma_{44}{ }^{*} S(P)(-1),
$$

The trace can be evaluated using (21)

$$
\begin{aligned}
& \int \frac{d^{4} p}{(2 \pi)^{4}} \operatorname{tr}^{*} S(P)\left[\Gamma_{4}\left(P, P^{\prime}\right)-\Gamma_{4}(P+Q, P)\right] \frac{1}{\varpi} \\
& \quad=\int \frac{d^{4} p}{(2 \pi)^{4}}\left[\operatorname{tr}^{*} S(P)^{*} \Gamma_{4}\left(P, P^{\prime}\right)-\operatorname{tr}^{*} S\left(P^{\prime}\right)^{*} \Gamma_{4}\left(P, P^{\prime}\right)\right] \frac{1}{\varpi},
\end{aligned}
$$

where in the second line, we make a change of variable: $P \rightarrow P^{\prime}$ to the second $\Gamma_{4}$. This expression will be canceled by part of the terms in the quark-antiquark diagram.
We proceed by simplifying the quark-antiquark diagram using (21). Note that ${ }^{*} \Gamma_{4}^{5}={ }^{*} \Gamma_{4} \gamma^{5}$ and also ${ }^{*} \Gamma_{4}\left(P, P^{\prime}\right)=$ ${ }^{*} \Gamma_{4}\left(P^{\prime}, P\right)$, which is obvious from (16), and we have

$$
\begin{aligned}
& \int \frac{d^{4} P}{(2 \pi)^{4}} \operatorname{tr}^{*} S(P)^{*} \Gamma_{4}^{5}\left(P, P^{\prime}\right) * S\left(P^{\prime}\right)^{*} \Gamma_{4}^{5}\left(P^{\prime}, P\right)(-1) \\
& =\int \frac{d^{4} P}{(2 \pi)^{4}} \operatorname{tr} \frac{1}{\not P+\Sigma+m}{ }^{*} \Gamma_{4} \gamma^{5} \frac{1}{P^{\prime}+\Sigma^{\prime}+m}\left(\Sigma-\Sigma^{\prime}\right) \gamma^{5} \frac{1}{\varpi},
\end{aligned}
$$

where we use shorthand notation $\Sigma=\Sigma(P), \Sigma^{\prime}=\Sigma\left(P^{\prime}\right)$ and suppressed the argument of ${ }^{*} \Gamma_{4}$ for notational simplicity. Commuting $\gamma^{5}$ through only the switch sign of mass in the second propagator, we can further simplify the expression by splitting $\Sigma-\Sigma^{\prime}=(\not P+\Sigma+m)-\left(\not \boldsymbol{P}^{\prime}+\Sigma^{\prime}-m\right)-$ $\left(\not P-\not P^{\prime}+2 m\right)$ and using the cyclic property of trace to obtain

$$
\begin{aligned}
& \int \frac{d^{4} P}{(2 \pi)^{4}} \operatorname{tr} \frac{1}{\not P+\Sigma+m}{ }^{*} \Gamma_{4} \frac{1}{\not P^{\prime}+\Sigma^{\prime}-m}\left(\Sigma-\Sigma^{\prime}\right) \frac{1}{\varpi} \\
& =\int \frac{d^{4} P}{(2 \pi)^{4}}\left[\operatorname{tr} \frac{1}{\not^{\prime}+\Sigma^{\prime}-m}{ }^{*} \Gamma_{4}-\frac{1}{\not P+\Sigma+m}{ }^{*} \Gamma_{4}\right. \\
& \left.-\operatorname{tr} \frac{1}{\not P+\Sigma+m}{ }^{*} \Gamma_{4} \frac{1}{\not^{\prime}+\Sigma^{\prime}-m}\left(\not P-\not P^{\prime}+2 m\right)\right] \frac{1}{\varpi} .
\end{aligned}
$$

The retarded correlator is given by the sum of (23) and (25).

It is instructive to look at the result in the massless limit first. Setting $m=0$, we immediately see the first two terms of (25) cancel (23) entirely, leaving only the third term of (25). To evaluate the third term, we use (18) and the following explicit expression of $\Gamma_{4}$ :

$$
\begin{aligned}
{ }^{*} \Gamma_{4}\left(P, P^{\prime}\right)= & {\left[\left(1-\frac{m_{f}^{2}}{i \varpi p} \delta Q_{0}\left(p, p^{\prime}\right)\right) \gamma_{4}\right.} \\
& \left.+\frac{m_{f}^{2}}{\varpi} \delta Q_{1}\left(p, p^{\prime}\right) \gamma_{p}\right],
\end{aligned}
$$

with $\delta Q_{n}\left(P, P^{\prime}\right)=Q_{n}\left(\frac{i \omega}{p}\right)-Q_{n}\left(\frac{i \omega^{\prime}}{p}\right)$. We can adopt a representation of $\Gamma_{4}$ in terms of $\Delta_{ \pm} \equiv \Delta_{ \pm}(P)$ and $\Delta_{ \pm}^{\prime} \equiv$ $\Delta_{ \pm}\left(P^{\prime}\right)$ by using (19),

$$
\begin{aligned}
{ }^{*} \Gamma_{4}\left(P, P^{\prime}\right)= & -\frac{1 / \Delta_{+}+1 / \Delta_{-}-1 / \Delta_{+}^{\prime}-1 / \Delta_{-}^{\prime}}{2 \varpi} i \gamma_{4} \\
& +\frac{1 / \Delta_{+}-1 / \Delta_{-}-1 / \Delta_{+}^{\prime}+1 / \Delta_{-}^{\prime}}{2 \varpi} \gamma_{p} .
\end{aligned}
$$

Taking the trace, we obtain

$$
\int \frac{d^{4} P}{(2 \pi)^{4}} \frac{2 i}{\varpi}\left(\Delta_{-}+\Delta_{+}-\Delta_{-}^{\prime}-\Delta_{+}^{\prime}\right)
$$


which vanishes identically upon change of variable. This indicates that indeed the contribution we are after is intrinsic to the breaking of the axial symmetry.

Now we move on to a massive case. We note that the first two terms of (25) combine with (23) to give

$$
\int \frac{d^{4} P}{(2 \pi)^{4}} \operatorname{tr}^{*} \Gamma_{4}\left(\frac{1}{\not p^{\prime}+\Sigma^{\prime}-m}-\frac{1}{\not p^{\prime}+\Sigma^{\prime}+m}\right) \frac{1}{\varpi},
$$

which still vanishes upon taking the trace. The remaining terms are

$$
\int \frac{d^{4} P}{(2 \pi)^{4}}(-) \operatorname{tr} \frac{1}{\not P+\Sigma+m}{ }^{*} \Gamma_{4} \frac{1}{\not P^{\prime}+\Sigma^{\prime}-m}\left(\not P-\not P^{\prime}+2 m\right) \frac{1}{\varpi} .
$$

We aim at calculating the lowest order mass correction, which begins at order $O\left(\mathrm{~m}^{2}\right)$. It arises from the expansion of the denominator and the numerator of the propagators and mass term in $\Gamma_{4}$. It is easy to see that the expansion of the denominator still gives a vanishing result due to similar cancellation as the $O\left(\mathrm{~m}^{0}\right)$ result. The remaining correction can be organized as follows:

$$
\int \frac{d^{4} P}{(2 \pi)^{4}} \frac{8 m^{2}}{\varpi^{2}}\left(-\Delta_{+} \Delta_{-}-\Delta_{+}^{\prime} \Delta_{-}^{\prime}+\Delta_{+} \Delta_{-}^{\prime}+\Delta_{+}^{\prime} \Delta_{-}\right) .
$$

We use the following formula to perform the frequency sum:

$$
\begin{aligned}
& \operatorname{Im} T \Sigma_{n} g_{1}\left(i \omega_{n}\right) g_{2}\left(i\left(\omega_{n}-\omega\right)\right)=\pi\left(1-e^{\beta q_{0}}\right) \\
& \times \int_{-\infty}^{+\infty} \frac{d p_{0}}{2 \pi} \frac{d p_{0}^{\prime}}{2 \pi} \tilde{f}\left(p_{0}\right) \tilde{f}\left(p_{0}^{\prime}\right) \delta\left(q_{0}-p_{0}-p_{0}^{\prime}\right) \rho_{1}\left(p_{0}\right) \rho_{2}\left(-p_{0}^{\prime}\right) .
\end{aligned}
$$

Here $g_{1}$ and $g_{2}$ are two generic functions. $\rho_{1}$ and $\rho_{2}$ correspond to their spectral densities, $\rho_{1}=-2 \operatorname{Im} g_{1}$, $\rho_{2}=-2 \operatorname{Im} g_{2}$. Note that the analytic continuation $i \omega \rightarrow$ $q_{0}+i \eta$ is taken after the frequency sum and only the imaginary part of the result is kept in (32). The frequency sum of the first two terms in (31) gives a contribution with $q_{0}=0$, which vanishes identically due to the factor $1-e^{\beta q_{0}}$. The frequency sum of the remaining terms is given by

$$
\begin{aligned}
& \int \frac{d^{3} p}{(2 \pi)^{3}} \int \frac{d p_{0}}{2 \pi} \frac{d p_{0}^{\prime}}{2 \pi} \pi\left(1-e^{\beta q_{0}}\right) \tilde{f}\left(p_{0}\right) \tilde{f}\left(p_{0}^{\prime}\right) \delta\left(q_{0}-p_{0}-p_{0}^{\prime}\right) \frac{32 m^{2}}{q_{0}^{2}}\left(\operatorname{Im} \Delta_{+}\left(p_{0}\right) \operatorname{Im} \Delta_{-}\left(-p_{0}^{\prime}\right)+\operatorname{Im} \Delta_{-}\left(p_{0}\right) \operatorname{Im} \Delta_{+}\left(-p_{0}^{\prime}\right)\right) \\
& =\int \frac{d^{3} p}{(2 \pi)^{3}} \int \frac{d p_{0}}{2 \pi} \frac{d p_{0}^{\prime}}{2 \pi} \pi\left(1-e^{\beta q_{0}}\right) \tilde{f}\left(p_{0}\right) \tilde{f}\left(p_{0}^{\prime}\right) \delta\left(q_{0}-p_{0}-p_{0}^{\prime}\right) \frac{32 m^{2}}{q_{0}^{2}}\left(\operatorname{Im} \Delta_{+}\left(p_{0}\right) \operatorname{Im} \Delta_{+}\left(p_{0}^{\prime}\right)+\operatorname{Im} \Delta_{-}\left(p_{0}\right) \operatorname{Im} \Delta_{-}\left(p_{0}^{\prime}\right)\right) .
\end{aligned}
$$

We have used the property $\operatorname{Im} \Delta_{ \pm}\left(-p_{0}^{\prime}\right)=\operatorname{Im} \Delta_{\mp}\left(p_{0}^{\prime}\right)$ in the second line. Note that Wick rotation applies $N_{5}^{4} \rightarrow i N_{5}$, which gives an overall minus sign between the correlator of $N_{5}^{4}$ and the correlator of $N_{5}$. Using the Kubo-MartinSchwinger relation, we readily obtain

$$
\begin{aligned}
G_{G}^{>}\left(q_{0}\right)= & \int \frac{d^{3} p}{(2 \pi)^{2}} \int \frac{d p_{0}}{2 \pi} \frac{d p_{0}^{\prime}}{2 \pi} e^{\beta q_{0}} \tilde{f}\left(p_{0}\right) \tilde{f}\left(p_{0}^{\prime}\right) \\
& \times \delta\left(q_{0}-p_{0}-p_{0}^{\prime}\right) \frac{32 m^{2}}{q_{0}^{2}} \\
& \times\left(\operatorname{Im} \Delta_{+}\left(p_{0}\right) \operatorname{Im} \Delta_{+}{ }^{\prime}\left(p_{0}^{\prime}\right)+\operatorname{Im} \Delta_{-}\left(p_{0}\right) \operatorname{Im} \Delta_{-}^{\prime}\left(p_{0}^{\prime}\right)\right) .
\end{aligned}
$$

Note that we have identified the contribution with $G_{G}^{>}$ because it arises entirely from quark mass breaking of the axial symmetry. The spectral density appearing in (34) contains a contribution from poles and a cut. The convolution of two spectral densities gives rise to contributions from the following types: pole-pole, pole-cut, and cut-cut. A similar situation is encountered in the computation of soft dilepton production, showing a remarkable structure [41,42].

For the purpose of demonstrating late time dynamics of $N_{5}$, we focus on the small $q_{0}$ regime. In the limit $q_{0} \rightarrow 0$, we obtain $G_{G}^{>} \rightarrow \frac{4 \Gamma_{m}}{q_{0}^{2}}$, with $\Gamma_{m}$ defined as

$$
\begin{aligned}
\Gamma_{m}= & \int \frac{d^{3} p}{(2 \pi)^{2}} \frac{d p_{0}}{2 \pi} \frac{d p_{0}^{\prime}}{2 \pi} 8 m^{2} \delta\left(p_{0}+p_{0}^{\prime}\right) \tilde{f}\left(p_{0}\right) \tilde{f}\left(p_{0}^{\prime}\right) \\
& \times\left[\operatorname{Im} \Delta_{+}\left(p_{0}\right) \operatorname{Im} \Delta_{+}\left(p_{0}^{\prime}\right)+\operatorname{Im} \Delta_{-}\left(p_{0}\right) \operatorname{Im} \Delta_{-}\left(p_{0}^{\prime}\right)\right] .
\end{aligned}
$$

$\Gamma_{m}$ characterizes the rate of fluctuation of the axial charge. To see this, we do the Fourier transform as follows:

$$
\begin{aligned}
& \int d^{3} x\left\langle\left(n_{5}(t, x)-n_{5}(0)\right)^{2}\right\rangle \\
& =\int \frac{d q_{o}}{2 \pi}\left(2-e^{-i q_{0} t}-e^{i q_{0} t}\right) G^{>}\left(q_{0}\right) \\
& \simeq \int \frac{d q_{0}}{2 \pi} \frac{8 \Gamma_{m}}{q_{0}^{2}}\left(1-\cos q_{0} t\right)=4 \Gamma_{m} t .
\end{aligned}
$$


This is the random walk growth of axial charge, with the growth rate given by (35).

To evaluate $\Gamma_{m}$, we note that the delta function constraint only allows for the cut-cut contribution in the product $\operatorname{Im} \Delta_{ \pm} \operatorname{Im} \Delta_{ \pm}^{\prime}$ : The pole-pole contribution is excluded in the limit $q_{0} \rightarrow 0$. The pole-cut contribution is possible only at large $p$, which is exponentially suppressed by the FermiDirac distribution. The cut-cut contribution is not suppressed. We send $\tilde{f}\left(p_{0}\right) \rightarrow 1 / 2, \tilde{f}\left(p_{0}^{\prime}\right) \rightarrow 1 / 2$ in the evaluation. All the momenta are of order $g T$. The spectral functions scale as $\operatorname{Im} \Delta_{ \pm} \sim g T$. As a result, we obtain $\Gamma_{m} \sim m^{2} g^{2} T^{2}$. This is to be compared with the ChernSimons (CS) diffusion rate, which scales as $\Gamma_{\mathrm{CS}} \sim$ $g^{10} \ln g^{-1} T^{4}[43,44]$ or $\Gamma_{\mathrm{CS}} \sim g^{8} T^{4}$ from extrapolation of the weak coupling result [32]. At sufficient weak coupling, the quark mass diffusion rate always dominates the CS diffusion rate. It is interesting to compare the actual number of the two rates at relevant coupling and mass. For the former, we quote the strong coupling extrapolation by Moore and Tassler [32],

$$
\Gamma_{\mathrm{CS}} \sim 30 \alpha_{s}^{4} T^{4} .
$$

For the latter, we need to obtain the precise number in $\Gamma_{m} \sim m^{2}(g T)^{2}$ from (35). We obtain from numerical integration

$$
\Gamma_{m} \simeq 0.013 m^{2} m_{f}^{2} .
$$

We use strange quark mass $m=100 \mathrm{MeV}$ and use the lattice measured thermal mass [45], which is $m_{f} \simeq 1.0 T$. Taking $T=400 \mathrm{MeV}$ and $\alpha_{s}=0.3$ relevant for heavy ion collisions, we obtain

$$
\Gamma_{\mathrm{CS}} \simeq 0.24 T^{4}, \quad \Gamma_{m} \simeq 0.001 T^{4} .
$$

We found the quark mass effect is much less efficient in axial charge generation. However, the effect of the quark mass can be significantly enhanced when the temperature approaches the transition temperature from above. In this region, the relevant mass parameter is the constituent quark mass, which is enhanced by partial chiral symmetry breaking. ${ }^{2}$

Note that in the weakly coupled case, only the generation of the axial charge is obtained, and the damping effect is not visible. The reason can be understood by making an estimate of the damping timescale. Using the fluctuation-dissipation theorem, the damping timescale due to the quark mass is given by

$$
\tau_{m}=\frac{\chi T}{2 \Gamma_{m}},
$$

where $\chi$ is the axial charge susceptibility. To the leading order in $g$, it is given by the free theory result $\chi \sim g^{0} T^{2}$. We thus obtain $\tau_{m} \sim \frac{T}{m^{2} g^{2}}$. Obviously the relaxation is shorter for

\footnotetext{
${ }^{2}$ We thank Pengfei Zhuang for pointing this out for us. See also related work [46].
}

larger mass, consistent with the expectation on general grounds. Note that we assumed $m \sim g T$ in the calculation. The conjugate frequency to this timescale is $q_{0} \sim g^{4} T$, which lies well beyond the HTL regime.

\section{SUMMARY}

Let us compare the fluctuation of the axial charge in free theory and weakly coupled QGP. First of all, the fluctuations in both cases contain two contributions: one is proportional to susceptibility, originating from the charge exchange with a heat bath; the other contribution is intrinsic to the breaking of axial symmetry. Focusing on the contribution from the breaking of axial symmetry, we find that unlike the susceptibility term, quantum fluctuation is needed to give a nonvanishing contribution. In the case of free theory, the quantum fluctuation is provided by effective Pauli repulsion. This also explains the counterintuitive result we find: the fluctuation maximizes at zero temperature. It also implies that it is misleading to interpret this contribution as a correction to susceptibility. In the case of weakly coupled QGP, the fluctuation is given by quark-gluon interaction, which is enhanced by the presence of the thermal medium. The frequency dependence of the Wightman correlator of the two cases is given by the following:

$$
\begin{aligned}
\left\langle G_{G}^{>}\left(q_{0}\right)\right\rangle_{G}^{\text {rree }} & \sim \sqrt{q_{0}^{2}-4 m^{2}} \frac{m^{2}}{\left|q_{0}\right|} \theta\left(\left|q_{0}\right|-2 m\right), \\
\left\langle G_{G}^{>}\left(q_{0}\right)\right\rangle_{G}^{\text {QGP }} & \sim \frac{m^{2} m_{f}^{2}}{q_{0}^{2}} .
\end{aligned}
$$

In the free case, the Wightman correlator vanishes for $\left|q_{0}\right|<2 \mathrm{~m}$. This gives a flat asymptotic behavior for $\left\langle N_{5}^{2}(t)\right\rangle$ as shown in Fig. 2. In the weakly coupled QGP case, the Wightman correlator is nonvanishing, giving rise to random walk behavior for $\left\langle N_{5}^{2}(t)\right\rangle$ in the long time limit. Note that (41) is not applicable when $q_{0} \sim g^{2} T$. We expect the random walk growth of the axial charge to be cut off on an even longer timescale. In order to see the damping effect, we might need kinetic theory to access this timescale [42]. We leave it for future work.

\section{ACKNOWLEDGMENTS}

We are grateful to Kenji Fukushima, Lianyi He, Anping Huang, Rob Pisarski, and especially Pengfei Zhuang for insightful discussions. S. L. thanks Central China Normal University, Beihang University, Tsinghua University, Peking University, Institute of High Energy Physics for hospitality where part of this work is done. This work is in part supported by the Ministry of Science and Technology of China (MSTC) under the "973" Project No. 2015CB856904(4) (D. H.), by NSFC under Grants No. 11375070, No. 11735007, No. 11521064 (D. H.), by One Thousand Talent Program for Young Scholars (S. L.), and by NSFC under Grants No. 11675274 and No. 11735007 (S. L.). 
[1] A. Vilenkin, Equilibrium parity violating current in a magnetic field, Phys. Rev. D 22, 3080 (1980).

[2] D. Kharzeev, Parity violation in hot QCD: Why it can happen, and how to look for it, Phys. Lett. B 633, 260 (2006).

[3] D. Kharzeev and A. Zhitnitsky, Charge separation induced by $P$-odd bubbles in QCD matter, Nucl. Phys. A797, 67 (2007).

[4] K. Fukushima, D. E. Kharzeev, and H. J. Warringa, The chiral magnetic effect, Phys. Rev. D 78, 074033 (2008).

[5] K. Fukushima, D. E. Kharzeev, and H. J. Warringa, RealTime Dynamics of the Chiral Magnetic Effect, Phys. Rev. Lett. 104, 212001 (2010).

[6] D. T. Son and B.Z. Spivak, Chiral anomaly and classical negative magnetoresistance of Weyl metals, Phys. Rev. B 88, 104412 (2013).

[7] L. Adamczyk et al., Beam-Energy Dependence of Charge Separation along the Magnetic Field in $\mathrm{Au}+\mathrm{Au}$ Collisions at RHIC, Phys. Rev. Lett. 113, 052302 (2014).

[8] B. Abelev et al., Charge Separation Relative to the Reaction Plane in Pb-Pb Collisions at $\sqrt{s_{N N}}=2.76 \mathrm{TeV}$, Phys. Rev. Lett. 110, 012301 (2013).

[9] A. M Sirunyan et al. (CMS Collaboration), Constraints on the chiral magnetic effect using charge-dependent azimuthal correlations in $\mathrm{pPb}$ and $\mathrm{PbPb}$ collisions at the LHC, Phys. Rev. C 97, 044912 (2018).

[10] Q. Li, D. E. Kharzeev, C. Zhang, Y. Huang, I. Pletikosic, A. V. Fedorov, R. D. Zhong, J. A. Schneeloch, G. D. Gu, and T. Valla, Observation of the chiral magnetic effect in ZrTe5, Nat. Phys. 12, 550 (2016).

[11] J. Gooth et al., Experimental signatures of the mixed axialgravitational anomaly in the Weyl semimetal NbP, Nature (London) 547, 324 (2017).

[12] D. E. Kharzeev, J. Liao, S. A. Voloshin, and G. Wang, Chiral magnetic and vortical effects in high-energy nuclear collisions-A status report, Prog. Part. Nucl. Phys. 88, 1 (2016).

[13] J. Liao, Anomalous transport effects and possible environmental symmetry "violation" in heavy-ion collisions, Pramana 84, 901 (2015).

[14] X.-G. Huang, Electromagnetic fields and anomalous transports in heavy-ion collisions-A pedagogical review, Rep. Prog. Phys. 79, 076302 (2016).

[15] D. T. Son and P. Surowka, Hydrodynamics with Triangle Anomalies, Phys. Rev. Lett. 103, 191601 (2009).

[16] Y. Neiman and Y. Oz, Relativistic hydrodynamics with general anomalous charges, J. High Energy Phys. 03 (2011) 023.

[17] K. Landsteiner, E. Megias, and F. Pena-Benitez, Gravitational Anomaly and Transport, Phys. Rev. Lett. 107, 021601 (2011).

[18] D. T. Son and N. Yamamoto, Berry Curvature, Triangle Anomalies, and the Chiral Magnetic Effect in Fermi Liquids, Phys. Rev. Lett. 109, 181602 (2012).

[19] D. T. Son and N. Yamamoto, Kinetic theory with Berry curvature from quantum field theories, Phys. Rev. D 87, 085016 (2013).

[20] M. A. Stephanov and Y. Yin, Chiral Kinetic Theory, Phys. Rev. Lett. 109, 162001 (2012).
[21] S. Pu, J.-h. Gao, and Q. Wang, A consistent description of kinetic equation with triangle anomaly, Phys. Rev. D 83, 094017 (2011).

[22] J.-W. Chen, S. Pu, Q. Wang, and X.-N. Wang, Berry Curvature and Four-Dimensional Monopoles in the Relativistic Chiral Kinetic Equation, Phys. Rev. Lett. 110, 262301 (2013).

[23] N. Mueller and R. Venugopalan, The chiral anomaly, Berry's phase and chiral kinetic theory, from world-lines in quantum field theory, Phys. Rev. D 97, 051901 (2018).

[24] N. Mueller and R. Venugopalan, Worldline construction of a covariant chiral kinetic theory, Phys. Rev. D 96, 016023 (2017).

[25] H.-U. Yee, Holographic chiral magnetic conductivity, J. High Energy Phys. 11 (2009) 085.

[26] K. Landsteiner, E. Megias, L. Melgar, and F. Pena-Benitez, Holographic gravitational anomaly and chiral vortical effect, J. High Energy Phys. 09 (2011) 121.

[27] C. Hoyos, T. Nishioka, and A. O'Bannon, A chiral magnetic effect from AdS/CFT with flavor, J. High Energy Phys. 10 (2011) 084.

[28] S. Lin and H.-U. Yee, Out-of-equilibrium chiral magnetic effect at strong coupling, Phys. Rev. D 88, 025030 (2013).

[29] A. Jimenez-Alba, K. Landsteiner, and L. Melgar, Anomalous magnetoresponse and the Stückelberg axion in holography, Phys. Rev. D 90, 126004 (2014).

[30] A. Jimenez-Alba, K. Landsteiner, Y. Liu, and Y.-W. Sun, Anomalous magnetoconductivity and relaxation times in holography, J. High Energy Phys. 07 (2015) 117.

[31] Y. Wu, D. Hou, and H.-c. Ren, Field theoretic perspectives of the Wigner function formulation of the chiral magnetic effect, Phys. Rev. D 96, 096015 (2017).

[32] G. D. Moore and M. Tassler, The sphaleron rate in SU(N) gauge theory, J. High Energy Phys. 02 (2011) 105.

[33] Y. Akamatsu, A. Rothkopf, and N. Yamamoto, Non-Abelian chiral instabilities at high temperature on the lattice, J. High Energy Phys. 03 (2016) 210.

[34] I. Iatrakis, S. Lin, and Y. Yin, The anomalous transport of axial charge: Topological vs non-topological fluctuations, J. High Energy Phys. 09 (2015) 030.

[35] D. Grabowska, D. B. Kaplan, and S. Reddy, Role of the electron mass in damping chiral plasma instability in supernovae and neutron stars, Phys. Rev. D 91, 085035 (2015).

[36] E.-d. Guo and S. Lin, Quark mass effect on axial charge dynamics, Phys. Rev. D 93, 105001 (2016).

[37] E.-d. Guo and S. Lin, Quark mass correction to chiral separation effect and pseudoscalar condensate, J. High Energy Phys. 01 (2017) 111.

[38] L. D. McLerran, A chiral symmetry order parameter, the lattice and nucleosynthesis, Phys. Rev. D 36, 3291 (1987).

[39] P. Copinger, K. Fukushima, and S. Pu, Axial Ward identity and the Schwinger mechanism-Applications to the realtime chiral magnetic effect and condensates, arXiv: 1807.04416.

[40] J. P. Blaizot, E. Iancu, and A. Rebhan, Comparing different hard thermal loop approaches to quark number susceptibilities, Eur. Phys. J. C 27, 433 (2003). 
[41] E. Braaten, R. D. Pisarski, and T.-C. Yuan, Production of Soft Dileptons in the Quark-Gluon Plasma, Phys. Rev. Lett. 64, 2242 (1990).

[42] G. D. Moore and J.-M. Robert, Dileptons, spectral weights, and conductivity in the quark-gluon plasma, arXiv:hep-ph/ 0607172.

[43] P. B. Arnold, D. Son, and L. G. Yaffe, The hot baryon violation rate is $\mathrm{O}\left(\alpha_{w}^{5} T^{4}\right)$, Phys. Rev. D 55, 6264 (1997).
[44] P. B. Arnold, D. T. Son, and L. G. Yaffe, Effective dynamics of hot, soft non-Abelian gauge fields. Color conductivity and $\log (1 / \alpha)$ effects, Phys. Rev. D 59, 105020 (1999).

[45] F. Karsch and M. Kitazawa, Quark propagator at finite temperature and finite momentum in quenched lattice QCD, Phys. Rev. D 80, 056001 (2009).

[46] M. Ruggieri, G. X. Peng, and M. Chernodub, Chiral relaxation time at the crossover of quantum chromodynamics, Phys. Rev. D 94, 054011 (2016). 